\title{
Correspondence
}

\section{A CASE OF BUREAU-BARRIERE SYNDROME IN A PATIENT WITH PSORIASIS VULGARIS}

\author{
Hristo P Dobrev, MD PhD ${ }^{1}$, Penka A Atanassova, MD, PhD ${ }^{2 *}$ \\ and Borislav D Dimitrov, MD MSc PhD ${ }^{3}$
}
Department of ${ }^{1}$ Dermatology \& Veneorology, and ${ }^{2}$ Neurology, Medical University, Plovdiv, 4000, Bulgaria; ${ }^{3}$ Academic Unit of Primary Care \& Population Sciences, University of Southampton, Southampton, SO16 6YD, UK

Short title: Bureau-Barrière syndrome and psoriasis vulgaris

*Corresponding author:

Dr Penka A Atanassova, MD PhD

Associate Professor

Department of Neurology, Medical University

15A V. Aprilov Blvd., Plovdiv, 4000, Bulgaria

fax: +359-32 6499 39, e-mail: pp atanassova@yahoo.com

Words count: 594 (without title page and references)

Illustrations: 1 figure (panels $A \& B$ ) 


\section{Dear Editor,}

Bureau-Barrière syndrome (BBS) is a rare condition that can affect various organ systems. It had been first reported in the 50-ies of the last century by the French dermatologists Prof. Yves Bureau and Prof. Henri Barrière $[\mathbf{1}, \mathbf{2}]$. Difficult to diagnose, it has been described as "non-familial ulceromutilating acropathy" and has been defined by the triad of painless ulcers at mechanically irritated and hyperkeratotic plantar areas of the feet, sensitive polyneuropathy of the lower legs and osteolysis in the forefoot area. The condition is sporadic and mainly affects middle-aged men suffering from liver disease, obesity or diabetes mellitus as well as alcoholism. Differential diagnosis commonly includes Thevenard syndrome (acropathia ulceromutilans familiaris), syringomyelia and tabes dorsalis.

In Bulgaria, the first patient with BBS was described by Popov et al in 1963 (as cited in [3]); another 4 patients were also reported till now [3]. We report here another, very rare case of a Bulgarian patient with Bureau-Barrière syndrome and psoriasis vulgaris (PV).

Our male patient (aged 48) presented with a 10-year history of periodic appearances of small plantar ulcers (basis of I-st and V-th digits) which persisted despite the treatment applied. The history also revealed a diagnosis of psoriasis vulgaris since 28 years, several traumatic fractures of booth feet, left forearm and lumbal vertebres in the period 2000-2008, and a regular alcohol consumption (up to $300 \mathrm{ml}$ for 25 years), without any related family predisposition. The patient reported for excessive transpiration of the feet since early age.

The general status was normal. X-ray examination revealed lumbar osteoporosis, spondylosis and compression fractures of T11 and L1, foot osteoporosis and deformations, mostly on the left. The neurological examination revealed distal hypoesthesia with sensorimotor syndrome of polyneuropathy of the lower extremities. The results from the nerve conduction studies and sympathetic skin responses (not shown) indicated the presence of an axonal polyneuropathy. Doppler examination of the patient's lower limbs was normal. Laboratory studies including blood sugar and immunological tests were within normal range.

On dermatological examination foot deformity and several plantar ulcers (Figure 1A) of "collerette cornée" type with a diameter of $2 \mathrm{~cm}$, rugged margins and secreting fundus were observed. In the same time, erythema-papulo-squamous plaques on the face, scalp, behind ears, trunk and extremities were seen (Figure 1B). Also, yellowing, onychodystrophy as well as subungual hyperkeratosis on the 1-st digit of the foot are noticed. The microbiological investigation of the ulcers revealed Proteus vulgaris while the ungual mycological investigation revealed Rhodotorula and Trichophyton mentagrophites. 
Based on the above finding, the diagnosis of Bureau-Barrière syndrome ("acropathia ulceromutilans et deformans pseudosyringomyelitica non familiaris extremitatum inferiorum") was established. A complex treatment including local antiseptic and anti-psoriatic medications as well as systemic chemotherapeutic, vasodilators, antimycotic and polyvitamin medications was applied. As a result, an improvement was noticed just on the 7-th day after the start of treatment. During the follow-up examination made one year later, a fully epithelisation was observed. (Figure 1).

Our literature search found several titles with BBS being reported most frequently in the lower extremities, among alcoholics and also among diabetic patients. There are few reports about associations of BBS with other diseases or conditions. Momeni and Stark [4] reported a case of aggressive ulcerating squamous cell carcinoma (Marjolin's ulcer) in a patient with Bureau-Barrière syndrome, while the single case in a psoriatic patient till now was reported as an association of BBS with Launois-Bensaude syndrome [5].

In conclusion, we described the second case of Bureau-Barriere syndrome in association with psoriasis vulgaris. Timely recognition of this rare condition could improve individual prognosis and patient's quality of life.

\section{References}

1. Bureau $\mathrm{Y}$, Barriere $\mathrm{H}$. Ulcerating and mutilating trophic lesions of the lower limbs. Br J Dermatol 1958; 70: 372-7.

2. Bureau, Y., H. Barriere $\mathrm{H}$, et al. Nonfamilial pseudosyringomyelic ulceromutilating acropathies of the lower limbs; concerning 23 observations. Presse Med 1957; 65: 2127-32. (In French with English abstr.)

3. Dobrev H, Markova T. Bureau-Barriere syndrome. Dermatol Venerol (Sofia) 1992; 31: 31-4. (in Bulgarian with English abstr.)

4. Momeni A, Stark GB. Early Marjolin's ulcer in Bureau-Barriere syndrome. Int J Low Extrem Wounds 2006; 5: 204-6.

5. Preisz K, Karpati S, et al. Launois-Bensaude syndrome and Bureau-Barriere syndrome in a psoriatic patient: successful treatment with carbamazepine. Eur J Dermatol 2002; 12: 267-9. 


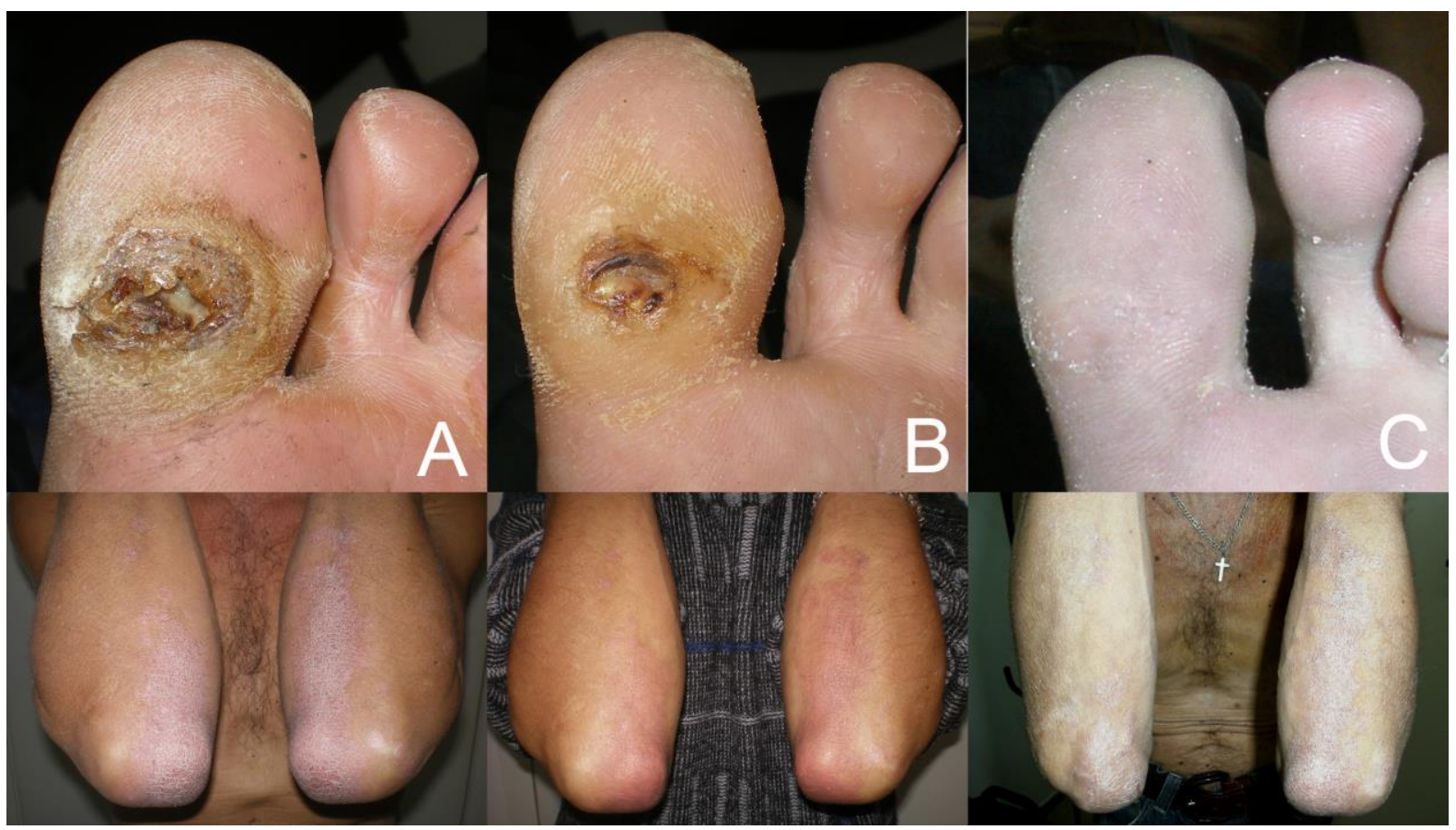

Figure 1 Dermatological symptoms in a patient with Bureau-Barriere syndrome and psoriasis vulgaris - (A) on admission; (B) after 7 days of treatment; (C) on the follow-up examination (C). 